\title{
Analytical Force and Flux for a One-Dimensional Electromagnetic Vibration Energy Harvester
}

Imbaquingo Muñoz, Carlos Enrique; Beleggia, Marco; Insinga, Andrea Roberto; Bahl, Christian; Mann, Brian; Bjørk, Rasmus

Published in:

IEEE Transactions on Magnetics

Link to article, DOI:

10.1109/TMAG.2020.3024734

Publication date:

2020

Document Version

Peer reviewed version

Link back to DTU Orbit

Citation (APA):

Imbaquingo Muñoz, C. E., Beleggia, M., Insinga, A. R., Bahl, C., Mann, B., \& Bjørk, R. (2020). Analytical Force and Flux for a One-Dimensional Electromagnetic Vibration Energy Harvester. IEEE Transactions on Magnetics, 56(11), [8000906]. https://doi.org/10.1109/TMAG.2020.3024734

\section{General rights}

Copyright and moral rights for the publications made accessible in the public portal are retained by the authors and/or other copyright owners and it is a condition of accessing publications that users recognise and abide by the legal requirements associated with these rights.

- Users may download and print one copy of any publication from the public portal for the purpose of private study or research.

- You may not further distribute the material or use it for any profit-making activity or commercial gain

- You may freely distribute the URL identifying the publication in the public portal 


\title{
Analytical Force and Flux for a One-Dimensional Electromagnetic Vibration Energy Harvester
}

\author{
Carlos Imbaquingo ${ }^{1}$, Marco Beleggia ${ }^{2}$, Andrea R. Insinga ${ }^{1}$, Christian Bahl ${ }^{1}{\text { Brian } \text { Mann }^{3} \text {, and Rasmus Bjørk }}^{1}$ \\ ${ }^{1}$ Department of Energy Conversion and Storage, DTU Energy, Kongens Lyngby, 2800 Denmark \\ ${ }^{2}$ National Center for Nanofabrication and Characterization, DTU Nanolab, Kongens Lyngby, 2800 Denmark \\ ${ }^{3}$ Department of Mechanical Engineering and Materials Science, Duke University, Durham NC, 27708 USA
}

\begin{abstract}
A Vibration Energy Harvester (VEH) converts the kinetic energy of a moving source into electrical energy. Here we consider a one-dimensional electromagnetic vibration energy harvester (1D-EMVEH) which consists of three coaxial cylindrical permanent magnets enclosed in a tube, such that the middle magnet is levitating. The resulting movement of the middle magnet can then induce an electromotive force (emf) in one or more surrounding coils. Using an analytical model, we derive expressions for the 1D-EMVEHs characteristic frequency and output power by using Fourier space approach. First, the magnetostatic energy of the system as a function of the position of the levitating magnet is calculated. Its spatial gradient gives the force acting on a magnet, which drives its dynamics. Next, more accurate magnetic flux and emf expressions are obtained. The results are compared with experimental measurements, revealing an excellent agreement.
\end{abstract}

\section{Index Terms-Electromagnetic Energy Harvester, Magnetic Energy, Magnetic Flux, Fourier Space.}

\section{INTRODUCTION}

$\mathbf{T}$ HE term energy harvesting is nowadays used to describe the energy conversion process from different sources at small scale. Such sources can be heat, radiation, or motion, and the conversion technique can vary depending on the application. Here, the energy from vibrations, which can be harvested electromagnetically (EM) or using piezoelectric materials, is considered. It has been shown that for acoustic vibrations electromagnetic harvesters produce more power than piezoelectric harvesters [1]. A considerable number of electromagnetic energy harvesters have been developed [2], and have been used in wireless vibration sensors [3] or microgenerators [4]. When designing a vibration energy harvester (VEH) in view of specific applications it is important to take into account the range of frequencies, size, output power and nature of the vibration source. One particular type of electromagnetic VEH is the 1D-EMVEH, which consists of three coaxial cylindrical permanent magnets enclosed in a tube and facing each other with appropriate polarities so that the middle magnet keeps levitating [5]-[7]. This middle magnet can then vibrate through a set of coils where an electromotive force is induced. This design mitigates the mechanical friction that is present in a more classical design where the permanent magnet is mounted on an oscillating spring. However, a tube is still necessary, as Earnshaw's theorem prevents a stable equilibrium of the levitating magnet. In such a 1D-EMVEH system the interaction between the levitating permanent magnet and the coil(s) is equivalent to a damper, while the force between the magnets provides a restoring force. Previously, the force between the magnets has been approximated by a polynomial behavior [5], but this is only an approximation of the true force. Additionally, the electrical damping effect has been defined as a function of

Manuscript received August 13, 2020; revised September 14, 2020. Corresponding author: C. Imbaquingo (email: ceim@dtu.dk). the relative position between the permanent magnet and the coils [8]. However, the fact that the emf in each loop of the coil also depends on the relative position of the magnet has not been considered. In order to understand and predict the motion of the levitating magnet and thus the power that can be harvested, it is imperative to analyze the true force on the levitating magnet, resulting from both the permanent magnets and the force arising when the levitating magnet interacts with the coaxial coil(s).

A substantial number of different expressions have been derived for both the force between the permanent magnets as well as the induced electromotive force [9]. In this work, we derive the non-linear expressions of the 1D-EMVEH for the magnetic flux and induced electromotive force based on a Fourier transform approach. As these results will be shown in simple expressions involving only a single integral, which are much simpler than most previously derived expressions [9], [10]. A particular analysis is done to find the emf equation when the coil is below, around, and above the levitating magnet. The given model is validated by comparing with simulations and experimental results.

\section{Mathematical Model}

An electromagnetic vibration energy harvester can be described as a damped driven harmonic oscillator [11], where the levitating permanent magnet experiences a restoring force due to the fixed magnets, and a damping force from the interaction between the magnet and the coils, i.e. in essence the harvested power. The force on the levitating magnet from the two fixed magnets is often approximated with a spring force described by Hooke's law. A simple illustration of a 1D-EMVEH system is shown in Figure 1a. The equation of motion for the levitating magnet is: 


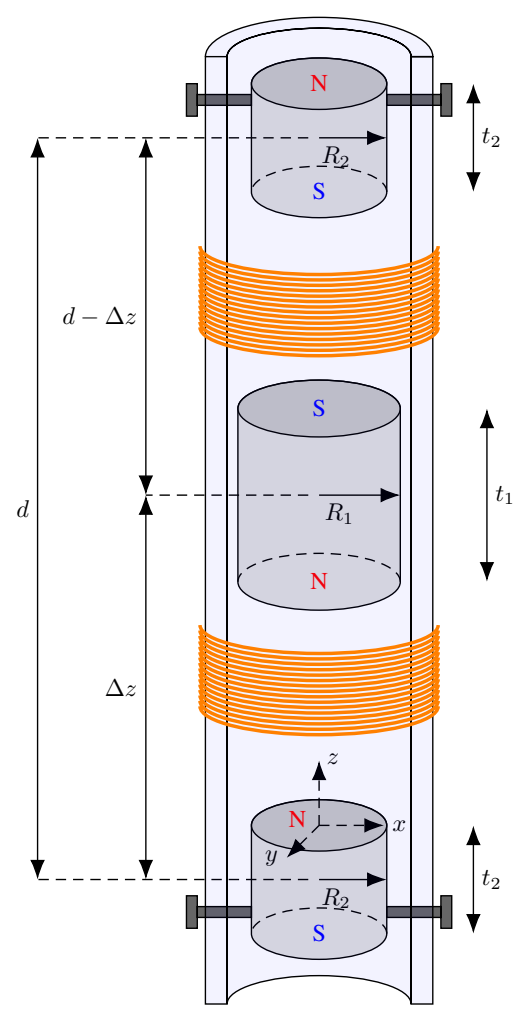

(a) Harvester basic schematic.

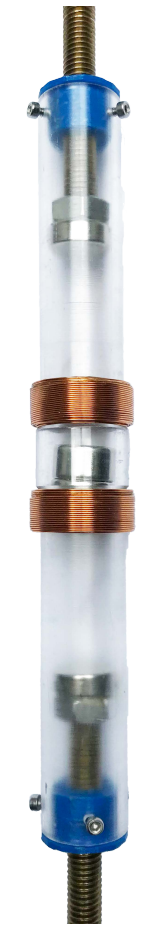

(b) Prototype.
Fig. 1: (a) Dimensions and relative position of the levitating (middle) and fixed (top and bottom) permanent magnets. (b) Prototype of One-dimensional Electromagnetic Vibration Energy Harvester 1D-EMVEH.

$$
m \frac{d^{2} z}{d t^{2}}+c \frac{d z}{d t}+k z+m g=F_{e x t}
$$

where $m$ is the mass of the levitating magnet, $g$ is the gravity acceleration, $F_{\text {ext }}$ is the external driving force, $k$ is the spring constant, related to the harvester's resonant frequency, and $c$ is the damping constant, related to the generated power. The spring constant will depend on the size and strength of the permanent magnets in the setup.

\section{A. Harvester Characteristic Frequency}

To solve the equation of motion, the spring constant $k$ and the damping coefficient $c$ are needed. The spring constant can be determined from the force between the permanent magnets in the system, which is the spatial gradient of its magnetostatic energy [12],

$$
E_{m}=-\frac{\mu_{o}}{2} \int_{V} \mathbf{H}(\mathbf{r}) \cdot \mathbf{M}(\mathbf{r}) d^{3} \mathbf{r}
$$

where $\mathbf{H}, \mathbf{M}, \mathbf{r}, \mu_{o}$ and $V$ are the magnetic field strength, magnetization, vector position in the Cartesian space, permeability in free space and volume respectively.

Assuming that the magnets are cylindrical, the energy can be determined from a mathematical analysis of the system, using the concept of the shape function $D(\mathbf{r})$, which is a discontinuous function equaling unity inside the object and zero outside [13], and which can be represented within Fourier space. The only assumption is that the magnets have a relative permeability of 1 , a value very close to the experimental value which is usually between 1.03-1.17 [14]. Previous derivations have considered a similar co-axial system of magnets as it is done here, but limited the analysis to magnets with identical dimensions [15], [16]. Using the same analytical principle, it is here considered the case where the magnet dimensions are not identical. To attain this modified expression, the derivation starting point contemplates the magnetic density (4), magnetization (5) and magnetic field strength (6) in Fourier space following [17].

$$
\begin{aligned}
\mathbf{B}(\mathbf{r}) & =\mu_{o}(\mathbf{M}(\mathbf{r})+\mathbf{H}(\mathbf{r})) \\
\mathbf{B}(\mathbf{k}) & =\frac{B_{r}}{k^{2}} D(\mathbf{k})\left(\hat{\mathbf{m}} k^{2}-\mathbf{k}(\hat{\mathbf{m}} \cdot \mathbf{k})\right) \\
\mathbf{M}(\mathbf{k}) & =M_{r} D(\mathbf{k}) \hat{\mathbf{m}} \\
\mathbf{H}(\mathbf{k}) & =-\frac{1}{k^{2}} \mathbf{k}(\mathbf{M}(\mathbf{k}) \cdot \mathbf{k})
\end{aligned}
$$

where $B_{r}=\mu_{o} M_{r}$ is the residual induction [18]. For the case of two bodies, the resulting magnetic field strength and magnetization are the sum of each body's contribution.

$$
\begin{aligned}
& \mathbf{M}(\mathbf{r})=\mathbf{M}_{1}\left(\mathbf{r}-\mathbf{r}_{1}\right)+\mathbf{M}_{2}\left(\mathbf{r}-\mathbf{r}_{2}\right) \\
& \mathbf{H}(\mathbf{r})=\mathbf{H}_{1}\left(\mathbf{r}_{1}\right)+\mathbf{H}_{2}\left(\mathbf{r}_{2}\right)
\end{aligned}
$$

and in Fourier space,

$$
\begin{aligned}
& \mathbf{M}(\mathbf{k})=\sum_{n=1}^{2} M_{n} D_{n}(\mathbf{k}) \hat{\mathbf{m}}_{n} e^{-i \mathbf{k} \cdot \mathbf{r}_{n}} \\
& \mathbf{H}(\mathbf{k})=-\frac{1}{k^{2}} \sum_{n=1}^{2} \mathbf{k}\left(\mathbf{M}_{n}(\mathbf{k}) \cdot \mathbf{k}\right)
\end{aligned}
$$

Thus the magnetostatic energy becomes (2).

$$
\begin{aligned}
E_{m}=\frac{\mu_{o}}{16 \pi^{3}} & \int \frac{d^{3} \mathbf{k}}{k^{2}}\left[\sum_{n=1}^{2}\left(\mathbf{M}_{n}(\mathbf{k}) \cdot \mathbf{k}\right)^{2}\right. \\
& +\left(\mathbf{M}_{1}(\mathbf{k}) \cdot \mathbf{k}\right)\left(\mathbf{M}_{2}(\mathbf{k}) \cdot \mathbf{k}\right) e^{i \mathbf{k} \cdot\left(\mathbf{r}_{2}-\mathbf{r}_{1}\right)} \\
& \left.+\left(\mathbf{M}_{2}(\mathbf{k}) \cdot \mathbf{k}\right)\left(\mathbf{M}_{1}(\mathbf{k}) \cdot \mathbf{k}\right) e^{-i \mathbf{k} \cdot\left(\mathbf{r}_{2}-\mathbf{r}_{1}\right)}\right]
\end{aligned}
$$

The first term of (11) is the self magnetic energy $E_{m, a}$ and the other terms represent the energy interaction between two 
bodies $E_{m, b}$.

$$
E_{m, b}=\frac{\mu_{o}}{8 \pi^{3}} \int \frac{d^{3} \mathbf{k}}{k^{2}}\left(\mathbf{M}_{1}(\mathbf{k}) \cdot \mathbf{k}\right)\left(\mathbf{M}_{2}(\mathbf{k}) \cdot \mathbf{k}\right) e^{i \mathbf{k} \cdot\left(\mathbf{r}_{2}-\mathbf{r}_{1}\right)}
$$

For the harvester of Figure 1, one fixed magnet and the floating cylindrical magnet represents the bodies in (12) whose shape function was derived by [15] and is expressed in (13).

$$
D(\mathbf{k})=\frac{2 \pi t R}{k_{\perp}} J_{1}\left(k_{\perp} R\right) \operatorname{sinc}\left(\frac{t k_{z}}{2}\right)
$$

where $t$ and $R$ are the length and radius of a cylindrical magnet, $k_{\perp}$ and $k_{z}$ are the components of the wave number vector $\mathbf{k}$, and $J_{1}$ is the Bessel's function of first order. The final magnetostatic interaction energy is the sum of the energy between the bottom magnet and the levitating magnet, where the ratio $\xi_{1}=\Delta z / R_{1}$, and between the top magnet and the levitating magnet whose ratio $\xi_{2}=(d-\Delta z) / R_{2}$. The result is given in (14).

$$
\begin{gathered}
E_{m, b i}=K_{e} R_{1}^{2} \int_{0}^{\infty} \frac{J_{1}(q) J_{1}\left(q R_{2} / R_{1}\right)}{q^{2}} \sinh \left(\tau_{1} q\right) \\
\cdot \sinh \left(\frac{R_{2}}{R_{1}} \tau_{2} q\right) e^{-\xi_{i} q} d q
\end{gathered}
$$

where $K_{e}=4 \pi \mu_{o} M_{1} M_{2} R_{2}, t_{i}=2 R_{i} \tau_{i}$ and $q=R_{2} / k_{\perp}$ is an equivalent integration variable, as shown in [19]. Subsequently, the repulsive magnetic force between sets of magnets, represented in (15), is derived as the negative gradient of the magnetic energy $\boldsymbol{F}_{m, i}=-\nabla E_{m, b i}$. Its solution depends on the position $\Delta z$ and the force will be oriented along the $\mathrm{z}$-axis.

$$
\begin{gathered}
F_{m, i}=K_{e} R_{1} \int_{0}^{\infty} \frac{J_{1}(q) J_{1}\left(q R_{2} / R_{1}\right)}{q} \sinh \left(\tau_{1} q\right) \\
\cdot \sinh \left(\frac{R_{2}}{R_{1}} \tau_{2} q\right) e^{-\xi_{i} q} d q
\end{gathered}
$$

Note that this force expression only contains a single integral, which numerically only has to be evaluated up to a cut-off value, and is thus easier to evaluate than the equally correct expression for the force derived by [20], which depends on six elliptic integrals.

Similarly, the total force of the system is the sum of the two resulting repulsive forces and the weight of the levitating magnet. At the equilibrium point $z_{e}$, the total force acting on the levitating magnet is equal to zero and represented in (16).

$$
\sum F\left(z_{e}\right)=F_{m 1}-F_{m 2}-F_{w}=0
$$

Also, around the equilibrium point, the system can be approximated to a simple harmonic oscillator, and the spring constant is found by evaluating the derivative of (17) at the equilibrium point $z_{e}$ shown in (18).

$$
\begin{gathered}
F_{T}=-k z \\
k=-\left.\frac{d F_{T}}{d z}\right|_{z_{e}}
\end{gathered}
$$

As a result, (19) is obtained, and the harvester characteristic frequency is found in (20).

$$
\begin{gathered}
k=K_{e} \int_{0}^{\infty} J_{1}(q) J_{1}\left(\frac{R_{2}}{R_{1}} q\right) \sinh \left(\tau_{1} q\right) \\
\cdot \sinh \left(\frac{R_{2}}{R_{1}} \tau_{2} q\right)\left(e^{-\xi_{e_{1}} q}-e^{-\xi_{e_{2}} q}\right) d q \\
f=\frac{1}{2 \pi} \sqrt{\frac{k}{m}}
\end{gathered}
$$

The force is linear as long as the distance between the levitating magnet and either of the end magnets is sufficiently large. This is always desirable in a harvester, as the levitating magnet must not touch the top or bottom magnets, as this could break the device.

\section{B. Damping Coefficient}

The damping of the motion of the magnet in an electromagnetic harvester is caused by the friction of the levitating permanent magnet with the inner tube walls and air, and importantly the repulsive (when the magnet is getting close to the coil) or attractive (when the magnet is getting away from the coil) magnetic force due to the current induced in the coil(s). The damping coefficient $c$ in (1) can be expressed according to [21] by

$$
c=c_{l}+\frac{\gamma^{2}}{R_{e q}}
$$

where $c_{l}$ represents the losses due to mechanical friction, $R_{e q}$ is the equivalent electrical resistance, and $\gamma$ is the damping coefficient due to the electromagnetic interaction. The latter is a function of the position [8], and related to the generated power. The power from the damping force, $P_{d}$, is the product of the damping force $F_{d}$, brought about by the electromagnetic interaction between the coil(s) and levitating magnet, and the speed of the magnet $v_{z}=d z / d t$. This must equal the generated electrical power, $P_{e}$, which is the product of the electromotive force emf and the electric current $I$, which depends on the resistance $R_{e q}$, i.e.

$$
P_{d}=F_{d} \cdot v_{z}=e m f \cdot I=P_{e}
$$

The damping force is given by the second term of (21) times the magnet speed as in (23), and the electromotive force is 
defined in (24). Consequently, the electromagnetic damping coefficient can be found as in (25).

$$
\begin{aligned}
F_{d} & =v_{z} \gamma^{2} / R_{e q} \\
e m f & =-v_{z} d \phi / d z \\
\gamma & =\frac{d \phi}{d z}
\end{aligned}
$$

where $\phi$ is the magnetic flux through the coil as a function of its position. Thus, in order to determine the damping coefficient, the flux from the levitating magnet through the coil must be known. Two situations must be considered, one where the magnet is inside the coil and one where it is outside. In the former situation, with the coil around the permanent magnet, the magnetic flux density is $\boldsymbol{B}=\mu_{o}(\boldsymbol{M}+\boldsymbol{H})$, since the magnetization of the levitating magnet contributes to the flux. This equation can also be expressed in Fourier space following [17] with the result shown in (4), and its corresponding inverse Fourier transform in (26). When the coil is above or below the magnet, the above equation simplifies to $\boldsymbol{B}=\mu_{o} \boldsymbol{H}$ hence (27) is attained. This situation is illustrated in Figure 2.

$$
\begin{aligned}
\mathbf{B}_{\text {in }} & =\frac{B_{r}}{8 \pi^{3}} \int d^{3} \mathbf{k} D(\mathbf{k})\left[\hat{\mathbf{m}}-\mathbf{k} \frac{\hat{\mathbf{m}} \cdot \mathbf{k}}{k^{2}}\right] e^{i \mathbf{k} \cdot \mathbf{r}} \\
\mathbf{B}_{\text {out }} & =-\frac{B_{r}}{8 \pi^{3}} \int \frac{d^{3} \mathbf{k}}{k^{2}} D(\mathbf{k}) \mathbf{k}(\hat{\mathbf{m}} \cdot \mathbf{k}) e^{i \mathbf{k} \cdot \mathbf{r}}
\end{aligned}
$$

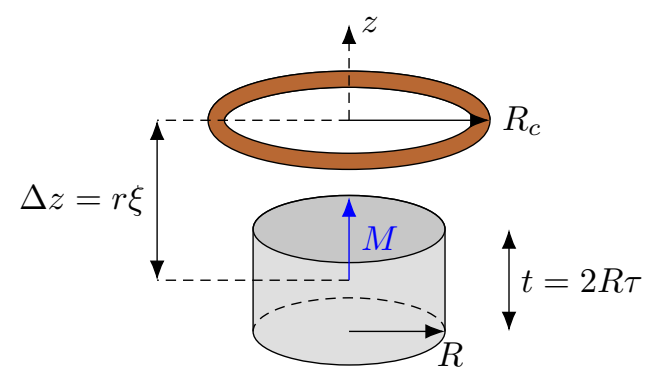

Fig. 2: Dimensions and position of the permanent magnet and coil.

As the levitating magnet and the coil are centered on the same axis, from symmetry it is only the z-component of the B-field that generates an inducing magnetic flux $\phi$. This flux is given in (28), assuming that the coil can be approximated as a geometric circle, i.e. with no height and defined by a single radius, $R_{c}$. Computing this in Fourier space, and afterwards evaluating the inverse Fourier transform gives the magnetic flux for the two configurations, i.e. with the magnet inside, (29), and outside, (30), the coil. These expressions match on the boundary when the magnet is about to the enter the coil.
The parameters of these two expressions are represented in Figure 2.

$$
\begin{gathered}
\phi=\int_{0}^{R_{c}} \rho d \rho \int_{0}^{2 \pi} d \theta B_{z}(\rho, \theta, z) \\
\phi_{\text {in }}=K_{f} R \int_{0}^{\infty} \frac{d q}{q} J_{1}(q) J_{1}\left(q R_{c} / R\right)\left(1-\cosh (q \xi) e^{-q \tau}\right) \\
\phi_{\text {out }}=K_{f} R \int_{0}^{\infty} \frac{d q}{q} J_{1}(q) J_{1}\left(q R_{c} / R\right) \sinh (q \tau) e^{-q \xi}
\end{gathered}
$$

where $K_{f}=2 \pi \mu_{o} M_{r} R_{c}$. The derivative of the flux with respect to time gives the electromotive force $e m f=-d \phi / d t$. This can also be expressed as the product of the permanent magnet speed and the derivative of flux with respect to position, $e m f=-v_{z} d \phi / d z$. The results of performing this calculation are shown in (31) and (32) for the induced voltage in a one-loop coil.

$$
\begin{gathered}
e m f_{\text {in }}=K_{f} v_{z} \int_{0}^{\infty} d q J_{1}(q) J_{1}\left(q R_{c} / R\right) \sinh (q \xi) e^{-q \tau} \\
e m f_{\text {out }}=K_{f} v_{z} \int_{0}^{\infty} d q J_{1}(q) J_{1}\left(q R_{c} / R\right) \sinh (q \tau) e^{-q \xi}
\end{gathered}
$$

To find the emf in a N-loop coil, an accepted approximation is to multiply the results of (31) and (32) by the number of coil turns $N$ as in [8]. One can also consider the full coil's stationary equivalent magnet [20], and calculate the force, $F_{d}$ between this and the moving magnet. If this force is known, the damping coefficient can be determined from (22) and (24) directly as

$$
\gamma=F_{d} / I
$$

where $I$ is the current in the coil. Equation (33) is essentially equal to (25), but [8] and [20] do not consider the position dependence of the flux with respect to each loop-coil. Therefore for both of the above mentioned approaches a comparison with experimental data shows a mismatch because the emf is a position dependent function, thus the induced voltage of each loop-coil is different. A more accurate procedure is to sum the contribution of each loop-coil whose radius $R_{c}$ and ratio $\xi$ depend on the layer number and position of the coil respectively.

$$
e m f_{N-\text { coil }}=\sum_{i=R_{c}}^{R_{w}} \sum_{j=z_{b}}^{z_{t}} \operatorname{emf}\left(R_{i}, z_{j}\right)
$$

where $R_{c}$ is the radius of the inner loop, $R_{w}$ the radius of the outer loop, $z_{b}$ the position of the bottom loop and $z_{t}$ the position of the top loop. 


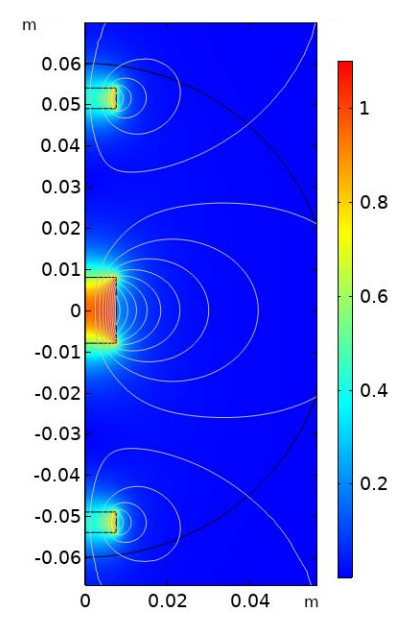

(a) Force

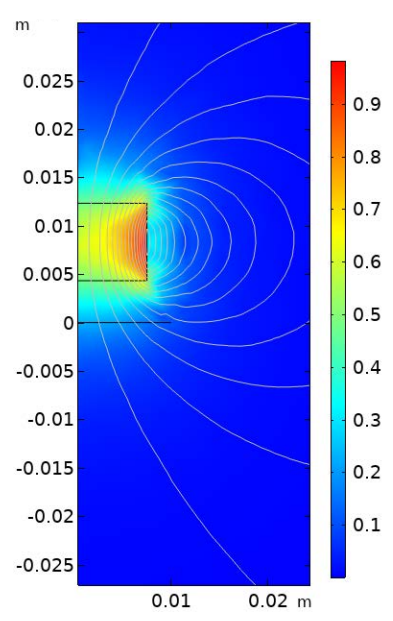

(b) Flux
Fig. 3: Magnetic flux density simulation results for the (a) magnetic force and (b) magnetic flux cases. The external vertical bar indicates the intensity of the magnetic flux density in tesla.

\section{Simulation MODEL}

The magnetic force and flux through a coil can also be obtained by simulating the magnetic field generated by the permanent magnets in, e.g., finite element method software. Here, the software COMSOL was used to verify the derived analytical expression by calculating the repulsive force that a levitating magnet experiences as function of its relative distance to the top and bottom magnets, as shown in Figure 3a. Furthermore, the magnetic flux from the levitating magnet through a one-loop coil was also calculated as illustrated in Figure 3b.

As it was mentioned in subsection II-A, the mathematical model uses the concept of a shape function, which basically equals one inside the object. The equivalence in the simulation model is to assume the magnetization is constant and distributed equally inside the permanent magnets. Then, the total flux on the N-loop coil is obtained by summing the contribution of each loop-coil as in subsection II-B, so the use of a fill factor as in [22] is avoided.

\section{EXPERIMENTAL VERIFICATION}

A 1D-EMVEH prototype was built to experimentally verify the derived expressions for the magnetic force and flux. The VEH consists of three parts: permanent magnets, acrylic tube and coils. The size of each component was selected according to commercial availability and the dimensions are detailed in Table I. The designed prototype harvester is shown in Figure 1b.

\section{A. Magnetic Force}

The experimental data were obtained by confining two permanent magnets in an acrylic tube, with the same magnetic
TABLE I: Dimensions and component specifications.

\begin{tabular}{ccc}
\hline Component & Parameter & Quantity \\
\hline \multirow{2}{*}{ Levitating magnet } & Radius & $7.5 \mathrm{~mm}$ \\
& Length & $16 \mathrm{~mm}$ \\
& Grade & NdFeB, N42 \\
\hline \multirow{2}{*}{ Fixed Magnets } & Radius & $7.5 \mathrm{~mm}$ \\
& Length & $8 \mathrm{~mm}$ \\
& Separation & $103 \mathrm{~mm}$ \\
& Grade & NdFeB, N42 \\
\hline \multirow{4}{*}{ Coils } & Inner radius & $10 \mathrm{~mm}$ \\
& Outer radius & $12.5 \mathrm{~mm}$ \\
& Length & $10 \mathrm{~mm}$ \\
& Separation & $15 \mathrm{~mm}$ \\
& Number of turns & 138 \\
& Wire gauge & $26 \mathrm{AWG}$ \\
& Resistance & $1.3 \Omega$ \\
& Inductance & $0.35 \mathrm{mH}$ \\
\hline
\end{tabular}

pole facing each other to keep the top magnet levitating by repulsion (see Figure 4). Next, known masses were placed on top of the levitating magnet to increase its weight and push it downwards. Then, the new distance between the two magnets was measured. The total mass, represented by the sum of the levitating magnet mass and the additional mass blocks, is multiplied by the gravitational acceleration so that the total resulting repulsive force is obtained.

The experimental, simulated and theoretical magnet force, as calculated by (15) is depicted in Figure 5, as a function of

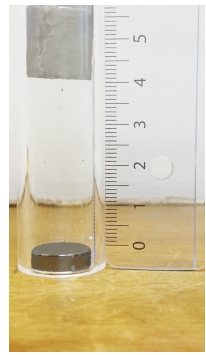

(a) No weight

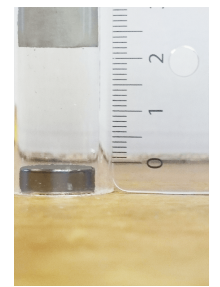

(b) mass $=110[\mathrm{~g}]$

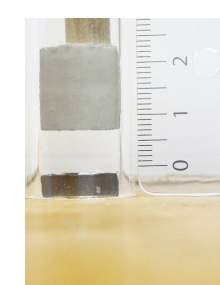

(c) mass $=934[\mathrm{~g}]$
Fig. 4: Position of the levitating magnet with different weights.

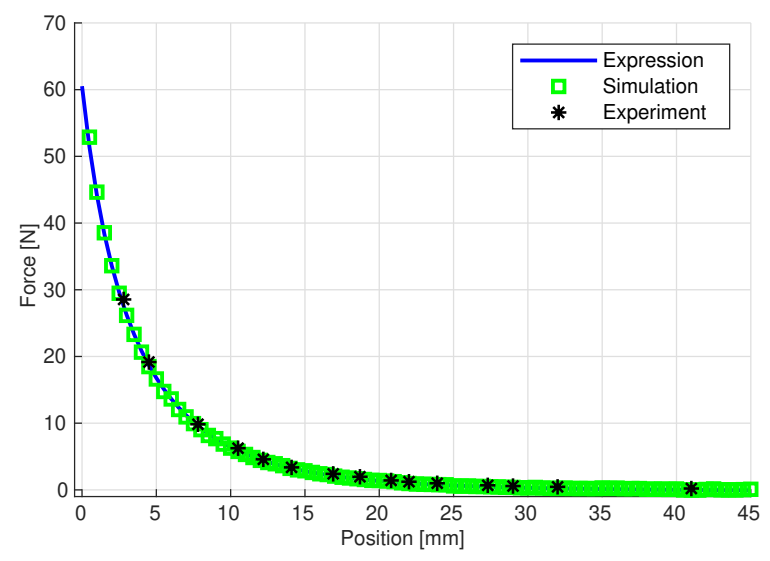

Fig. 5: The force on the levitating magnet as a function of its relative position in a configuration as given in Figure 1a. 
the distance between only the center magnet and the bottom magnet. As it can be seen, there is an excellent agreement between the derived expression for the force, the simulation results and the experimental measurement. This shows that (19) is an accurate approach to find the spring constant $k$, for the VEH. The solution of (19) gives $k=6.36 \mathrm{~N} / \mathrm{m}$ for this example.

\section{B. Gradient of the Magnetic Flux}

As the electromotive force given by (34) depends on the velocity of the permanent magnet passing through the coils, a setup is implemented and illustrated in Figure 6 so that such velocity is known.

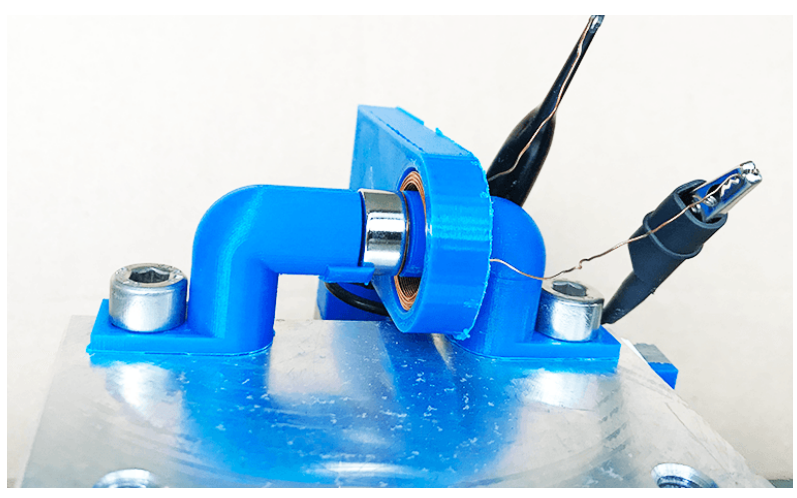

Fig. 6: Experimental setup to find the induced emf on a N-loop coil.

To validate the data, the experiment is carried out at three different frequencies, and the plotted results are the gradient of the flux as a function of the position. The induced voltage on the coil of Figure 6 is recorded and divided by the velocity given by a total horizontal displacement of $21 \mathrm{~mm}$. The gradient of the flux obtained from simulations is evaluated, and

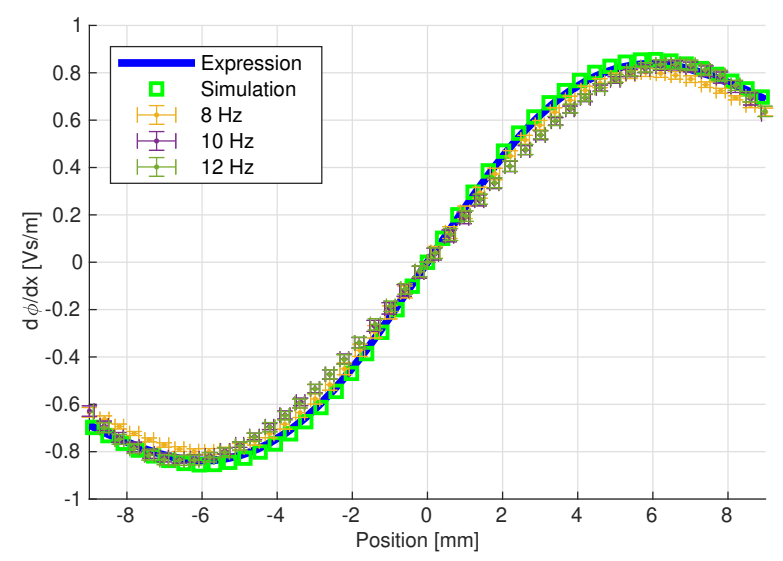

Fig. 7: Gradient of the magnetic flux with respect to the position in a N-loop coil when a cylindrical permanent magnet is passing through it. the correspondent gradient from the expression is found with (31) and (32), without the velocity being considered. Figure 7 depicts a really small mismatch due to the symmetrical way how the induced voltage on each loop-coil is summed to attain the total gradient of the flux on the N-loop coil with the mathematical expression and the simulation results. Part of the small discrepancy between the experimental and expression data can be caused by the fact that the magnets have a relative permeability slightly different from 1 [14].

\section{CONCLuSion}

In this paper, the mathematical expressions for the force between cylindrical axially magnetized permanent magnets, and the electromotive force on a coil placed co-axially with a permanent magnet were derived. These expressions were used to calculate the spring constant and the damping coefficient of a 1D electromagnetic vibrational energy harvester, in an easier manner because the analysis was done in Fourier space. The gradient of the energy and flux was reduced to a simple derivative of an exponential function. Next, the derived mathematical expressions were verified with both finite element simulations and experimental results, both of which showed excellent agreement with the expressions.

\section{ACKNOWLEDGMENT}

The authors wish to thank the Independent Research Fund Denmark, project 8022 00038B for sponsoring this work.

\section{REFERENCES}

[1] F. U. Khan and Izhar, "State of the art in acoustic energy harvesting," Journal of Micromechanics and Microengineering, vol. 25, no. 2, p. 023001, jan 2015. [Online]. Available: https://doi.org/10.1088\% 2F0960-1317\%2F25\%2F2\%2F023001

[2] C. Cepnik, R. Lausecker, and U. Wallrabe, "Review on electrodynamic energy harvesters - a classification approach," Micromachines, vol. 4, pp. 168-196, 2013.

[3] J. Qiu, X. Liu, H. Chen, X. Xu, Y. Wen, and P. Li, "A low-frequency resonant electromagnetic vibration energy harvester employing the halbach arrays for intelligent wireless sensor networks," IEEE Transactions on Magnetics, vol. 51, no. 11, pp. 1-4, Nov 2015.

[4] S. Saadon and O. Sidek, "Environmental vibration-based mems piezoelectric energy harvester (evmpeh)," in 2011 Developments in E-systems Engineering, Dec 2011, pp. 511-514.

[5] B. Mann and N. Sims, "Energy harvesting from the nonlinear oscillations of magnetic levitation," Journal of Sound and Vibration, vol. 319, no. 1, pp. 515 - 530, 2009. [Online]. Available: http://www.sciencedirect.com/science/article/pii/S0022460X08005567

[6] R. Morais, N. M. Silva, P. M. Santos, C. M. Frias, J. A. Ferreira, A. M. Ramos, J. A. Simoes, J. M. Baptista, and M. C. Reis, "Double permanent magnet vibration power generator for smart hip prosthesis," Sensors and Actuators A: Physical, vol. 172, no. 1, pp. 259 - 268, 2011, eurosensors XXIV, Linz, Austria, 5-8 September 2010. [Online]. Available: http://www.sciencedirect.com/science/article/pii/S0924424711002329

[7] G. D. Pasquale, A. Somà, and F. Fraccarollo, "Comparison between piezoelectric and magnetic strategies for wearable energy harvesting," Journal of Physics: Conference Series, vol. 476, p. 012097, dec 2013. [Online]. Available: https://doi.org/10.1088\%2F1742-6596\%2F476\% 2F1\%2F012097

[8] H. Lee, M. D. Noh, and Y. Park, "Optimal design of electromagnetic energy harvester using analytic equations," IEEE Transactions on Magnetics, vol. 53, no. 11, pp. 1-5, Nov 2017.

[9] P. Carneiro, M. P. S. dos Santos, A. Rodrigues, J. A. Ferreira, J. A. Simões, A. T. Marques, and A. L. Kholkin, "Electromagnetic energy harvesting using magnetic levitation architectures: A review," Applied Energy, vol. 260, p. 114191, 2020.

[10] A. A. Bernal and L. L. García, "The modelling of an electromagnetic energy harvesting architecture," Applied Mathematical Modelling, vol. 36, no. 10 , pp. $4728-4741,2012$. 
[11] R. Serway, Physics for scientists and engineers. Belmont, CA: Thomson-Brooks/Cole, 2004.

[12] M. Beleggia, S. Tandon, Y. Zhu, and M. D. Graef, "On the magnetostatic interactions between nanoparticles of arbitrary shape," Journal of Magnetism and Magnetic Materials, vol. 278, no. 1, pp. 270 - 284, 2004. [Online]. Available: http://www.sciencedirect.com/science/ article/pii/S0304885304000186

[13] M. Beleggia and M. D. Graef, "General magnetostatic shape shape interactions," Journal of Magnetism and Magnetic Materials, vol. 285, no. 1, pp. L1 - L10, 2005. [Online]. Available: http://www.sciencedirect.com/science/article/pii/S0304885304008923

[14] M. Katter, "Angular dependence of the demagnetization stability of sintered nd-fe-b magnets," IEEE Transactions on magnetics, vol. 41, no. 10, pp. 3853-3855, 2005.

[15] M. Beleggia and Y. Zhu, "Electron-optical phase shift of magnetic nanoparticles i. basic concepts," Philosophical Magazine, vol. 83, no. 8, pp. 1045-1057, 2003. [Online]. Available: https://doi.org/10. 1080/0141861031000066166

[16] D. Vokoun, M. Beleggia, L. Heller, and P. Šittner, "Magnetostatic interactions and forces between cylindrical permanent magnets," Journal of Magnetism and Magnetic Materials, vol. 321, no. 22, pp. 3758 - 3763, 2009. [Online]. Available: http://www.sciencedirect.com/ science/article/pii/S030488530900746X

[17] M. Beleggia and M. D. Graef, "On the computation of the demagnetization tensor field for an arbitrary particle shape using a fourier space approach," Journal of Magnetism and Magnetic Materials, vol. 263, no. 1, pp. L1 - L9, 2003. [Online]. Available: http://www.sciencedirect.com/science/article/pii/S0304885303002385

[18] MMPA, "Standard specifications for permanent magnet materials," Magnetic Materials Producers Association, Chicago, Illinois 60603, Standard, 1964.

[19] D. Vokoun, M. Beleggia, T. Rahman, H. C. Hou, and C. H. Lai, "The two-spin model with dipolar interactions for the exchange coupled composite media," Journal of Applied Physics, vol. 103, no. 7, p. 07F520, 2008.

[20] R. Ravaud, G. Lemarquand, S. Babic, V. Lemarquand, and C. Akyel, "Cylindrical magnets and coils: Fields, forces, and inductances," IEEE Transactions on Magnetics, vol. 46, no. 9, pp. 3585-3590, Sep. 2010.

[21] B. Mann and B. Owens, "Investigations of a nonlinear energy harvester with a bistable potential well," Journal of Sound and Vibration, vol. 329, no. 9, pp. 1215 - 1226, 2010. [Online]. Available: http://www.sciencedirect.com/science/article/pii/S0022460X0900978X

[22] S. P. Beeby and T. O'Donnell, Electromagnetic Energy Harvesting. Boston, MA: Springer US, 2009, pp. 129-161. [Online]. Available: https://doi.org/10.1007/978-0-387-76464-1_5 